\title{
Multi-objective Optimization for Many-to-Many Communication in Cognitive Radio Networks
}

\author{
Abdullah M. Almasoud and Ahmed E. Kamal, \\ Department of Electrical and Computer Engineering, Iowa State University, Ames, IA 50011, USA, \\ E-mails: \{almasoud, kamal\}@iastate.edu.
}

\begin{abstract}
In this paper, we investigate the problem of routing for supporting many-to-many communication and optimizing the Quality of Service (QoS) for the Secondary Users (SUs) in Cognitive Radio Networks (CRN). The goal is to build the routing tree for many-to-many communication that optimizes the delay, rate and the number of transmission links. We model the network using the Multi-Layer Hyper Graph, and we model the optimization problem as an Integer Linear Program (ILP). We also study the problem of many-to-many communication scheduling in CRNs. We employ a modified version of an Ant Colony optimization algorithm (MOACS) to solve our problem of finding the routing tree that optimizes our objectives. Our simulation results show that the performance of MOACS outperforms the Shortest-Path Tree (SPT) in all our objectives.
\end{abstract}

Index Terms-Many-to-many communication, group communication, cognitive radio network, scheduling, multi-objective optimization, ant colony system.

\section{INTRODUCTION}

Cognitive Radio (CR) is a promising technology, which enables dynamic sharing of the spectrum. In Cognitive Radio Networks (CRNs), Primary users (PUs) are licensed to use a certain part of the spectrum with a highest priority. On the other hand, Secondary Users (SUs) can access the licensed spectrum opportunistically when a PU is idle. By employing CR technology, spectrum scarcity problem in wireless networks can be alleviated by allowing the SUs to share unused parts of the spectrum.

With increasing demand of wireless networks, many-tomany communication arises as an essential service for many nowadays' applications. This communication paradigm has a wide range of applications, including video/audio conferencing, distributed gaming and cooperative processing. In manyto-many communication, the session consists of a group of users, where each user sends its messages to all other users in the group. To support a reliable many-to-many communication session, users' traffic is routed under certain minimum QoS requirements.

Provisioning a minimum cost many-to-many communication session is a hard problem, since finding the Steiner minimum tree [1] is NP-Hard problem [2]. Considering certain QoS requirements makes the problem harder. On the other hand, several challenges are also imposed as a result of the nature of CRNs. For example, channels availability for SUs in CRNs can be heterogeneous, which means that two SUs may have no common channel. Moreover, the SU may need to switch between different channels to receive and/or forward the traffic, and that imposes more delay which is called switching delay.

In this paper, we study multi-objective many-to-many communication optimization in CRNs. Given a many-to-many communication group, we establish a routing tree from each user to all other users in the group. We consider certain QoS requirements while provisioning the many-to-many communication tree, including delay minimization, data rate maximization and minimizing the number of used links in the network. The rest of paper is organized as follows. We introduce the system model in Section II. In Section III, we describe the multi-objective optimization problem for many-to-many communication in CRNs. Scheduling of SUs' transmission is described in Section IV, and we show in Section V how to solve the multi-objective optimization for many-to-many communication using Ant Colony Optimization. Finally, we discuss the simulation results in Section VI and conclude the paper in Section VII.

\section{SySTEM MODEL}

We consider a multi-hop cognitive radio network with $n$ Secondary Users (SUs). Each SU is equipped with a single radio and can access a single channel at a time either for transmission or reception. Channels availabilities for SUs are heterogeneous, i.e., the set of available channel for each user may vary depending on the location of the SU and the channel condition in its vicinity. SUs in the network use TV white space spectrum, where the spectrum activities are quasistatic.

The network is modeled using the Multilayer Hyper-Graph model introduced in [3]. The multilayer Hyper-Graph consists of a number of layers which represent the communication channels. Each layer (channel) has a set of Hyper-Edges (HE), and each HE consists of a set of SUs. Let $t=\left\{t_{1}, \cdots, t_{\max }\right\}$ be a set of transmission ranges, where $t_{\max }$ is the maximum transmission range. The SU can transmit to the other SUs with rate $r_{i}$ using a transmission range $t_{i}$, where $t_{1} \leq t_{i} \leq t_{\max }$. For each transmission range $t_{i}$, SUs using the same channel are grouped in one $\mathrm{HE}$ if all of them can transmit/receive to/from each other and within one hop. Therefore, the distance between any two SUs (say $S U_{j}$ and $S U_{k}$ ) located within one $\mathrm{HE}$ is no more than the transmission range within the $\mathrm{HE}$.

Given the transmission range, $t_{i}$, within an $\mathrm{HE}$, the upper bound on the transmission rate within the HE can be calculated using Shannon-Hartley's formula, which is given by

$$
C_{i}=W \log _{2}\left(1+\frac{P g}{t_{i}^{\epsilon} N_{0} W}\right)
$$


where $C_{i}$ is the channel capacity when the transmission range equals to $t_{i}, W$ is the channel bandwidth, $P$ is the transmission power, $g$ is the antenna gain, $N_{0}$ is the noise spectral density and $\epsilon$ is the path loss. The data rate of an HE depends on the transmission range of the SUs forming that HE. Let $\mathrm{HE} q$ be an $\mathrm{HE}$ where the transmission range of its corresponding SUs is $t_{k}$. The cost of the HE $\mathrm{q}$ is represented by transmission time of a packet within HE q, which is given by $\frac{\text { Packet size }}{C_{k}}$, where $C_{k}$ is the data rate of $\mathrm{HE} \mathrm{q}$.

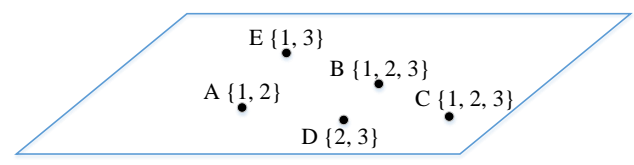

Fig. 1. SUs' locations and available channels.

Figure 1 shows a group of SUs and their channel assignments. In Figure 2, all SUs with a common channel are assigned to a layer, and the SUs located within the transmission range of each other are assigned to an HE. The rate of transmission inside HE's depends on the transmission range of the SU, where a shorter transmission range leads to a higher transmission rate. The transmission range of the SUs in HE1 and HE4 is $t_{1}$, in HE3 and HE6 is $t_{2}$ and in HE2 and HE5 is $t_{3}$. The rates corresponding to $t_{1}, t_{2}$ and $t_{3}$ are $r_{3}, r_{2}$ and $r_{1}$ respectively, where $t_{1}<t_{2}<t_{3}$ and $r_{3}<r_{2}<r_{1}$.

An SU may have more than one channel available as shown in Figure 2. The vertical dashed line represents switching delay when an SU receives a packet over a certain channel and forwards it over another channel. The switching delay between band $\mathrm{i}\left(W_{i}\right)$ and band $\mathrm{j}\left(W_{j}\right)$ is given by [4]:

$$
k\left|W_{i}-W_{j}\right|
$$

where $k$ is a technology dependent parameter, and we assume that $k=\frac{120 \mu \mathrm{s}}{75 \mathrm{MHz}}$ [5].

For each channel i, we assume that one Primary User (PU) can be active at a time and with a probability $P_{P U_{i}}$, where $P U_{i}$ is the PU licensed to channel i. Once $P U_{i}$ becomes active on channel $\mathrm{i}$, all SUs located within its transmission range will be blocked from accessing channel i. All HE's associated with these SUs are considered inactive when the PU becomes active.

Assume that there is a Many-to-Many communication request represented by SU A, SU B and SU C. Hence, it is required to route the traffic initiated by each of these SUs to all other SUs. The dummy nodes with $t$ and $r$ subscripts in Figure 2 represent sources and destinations of the Manyto-Many communication request, respectively. These dummy nodes are used to make source and destination SUs tune their radios to the channels that optimize our objective of minimizing the delay and the number of links and maximizing the data rate. The dummy links, which connect the dummy nodes to source/destination SUs, are virtual. Hence, we do not consider them in calculating the delay, number of links and the date rate.

Packets routing in cognitive radio networks can be over a single channel or multiple channels as needed. It is shown in

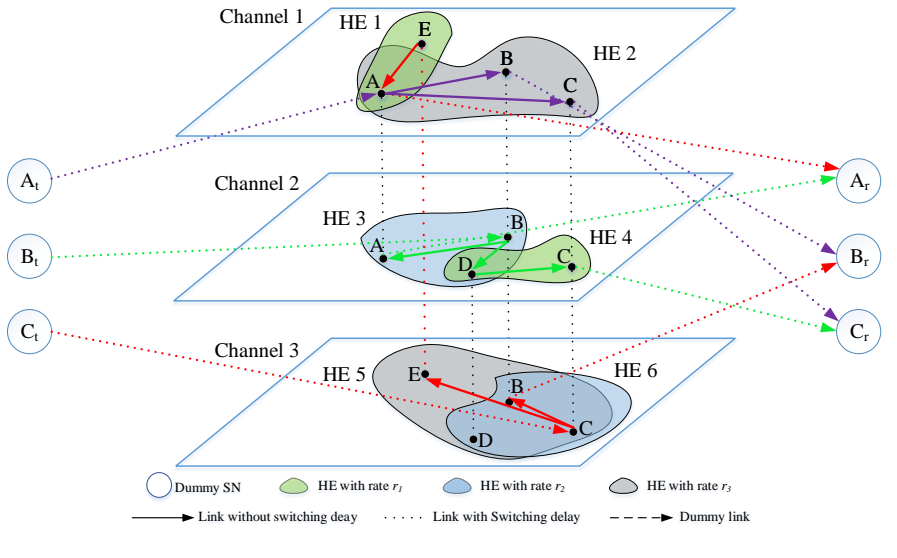

Fig. 2. Routing in Multilayers Hyper-Graph.

Figure 2 that all source SUs in the Many-to-Many communication session (A, B and C) belong to HE 2. Therefore, SU A can reach all other SUs in the session over channel 1 and within one hop. The dummy node, $A_{t}$, indicates that SU A needs to tune its radio over channel 1 for transmission, where the dummy nodes $B_{r}$ and $C_{r}$ indicate that they tune their radios to channel 1 to receive the messages from SU A. SU A has the choice of sending its messages with a higher date rate using channel 2. However, it will need one hop to reach SU B and two hops to reach SU C through SU D.

In contrast to $\mathrm{SU} A, \mathrm{SU} B$ can reach all destinations with a higher data rate, but with more hops. SU B can reach the other SUs in the session over channel 2 and with one hop to SU A (through HE 3) and two hops to SU C (through HE 3 and HE 4). SU C sends its messages over channel 3 through HE 5 , where SU B can rceive the messages over channel 3 and within one hop. However, SU A cannot operate on channel 3, and hence, SU E receives the traffic from SU C over channel 3 and switches to channel 1 to forward the traffic to SU A in HE 1. Spectrum switching performed by SU E to route SU C's traffic causes switching delay, which is represented by the dashed line between SU E on channel 1 and channel 3.

To construct the Many-to-Many communication subgraph, we convert the Multilayer Hyper-Graph to the mapped graph [3] as shown in Figure 3. Each HE is converted to a supernode (SN), which represents all SUs associated with an HE. An SN is equivalent to the HE, and we use both terms in this paper interchangeably. If there is an SU belonging to multiple HE's, then there is an overlap between these HE's. The overlap between two HE's means that an overlapping SU can forward the messages between the overlapping HE's. Therefore, any two overlapping HE's are represented by two super-nodes with a link connecting them as shown in Figure 3.

Let $V$ and $E$ be the sets of all SNs and all edges in the mapped graph respectively. Then, the mapped graph of the Multilayer Hyper-Graph is represented by $G(V, E)$. The solid lines in Figure 3 represent that both HEs at the end of the line belong to one layer, and hence, no switching delay is considered. However, if the overlapping HEs belong to different layers, then the super-nodes representing them are 
connected by a dashed line to reflect that a switching delay is considered in delay calculation.

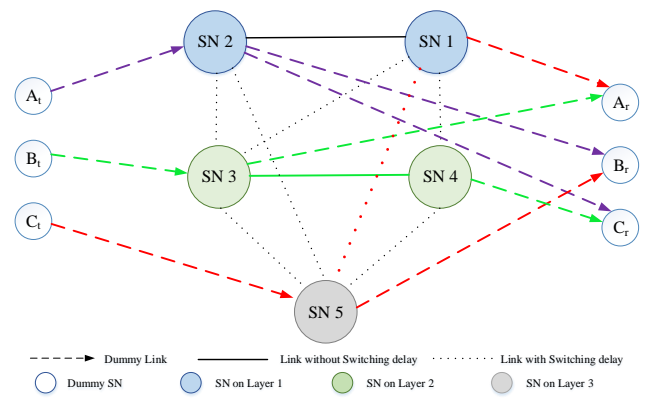

Fig. 3. Mapping the Multilayers Hyper-Graph.

\section{Multi-objective Optimization Problem for MANY-TO-MANY COMMUNICATION IN CRN}

The many-to-many session request is represented by a set of SUs, $S$, where each $\mathrm{SU} \in S$ sends/receives messages to/from all other SUs in the set. Establishing a minimum cost tree from an SU to all other SUs in the many-to-many session group is equivalent to finding the Steiner minimum tree [1], which is an NP-Hard problem [2]. We formulate our problem as an Integer Linear Program (ILP) as described in the following.

Let $S_{t}$ be a set of transmitting dummy nodes and $S_{r}$ be a set of receiving dummy nodes corresponding to the SUs in $S$. For an SU $p$, the transmitting and receiving dummy nodes corresponding to $p$ are denoted by $p_{t}$ and $p_{r}$ respectively. Let $M$ be a set of binary variables $M_{i j}^{p_{t} q_{r}}, \forall p_{t} \in S_{t}, \forall q_{r} \in$ $S_{r} \backslash p_{r}, \forall i, j \in V$, where $M_{i j}^{p_{t} q_{r}}$ is defined as

$$
M_{i j}^{p_{t} q_{r}}= \begin{cases}1, & \begin{array}{l}
\text { if there is a path from } p_{t} \text { to } q_{r} \text { using } \\
\text { link }(i, j)
\end{array} \\
0, & \text { otherwise }\end{cases}
$$

Our objectives are maximizing the date rate and minimizing the delay and the number of links that interconnect the SNs for a given Many-to-Many session request. Let $Y_{L}(M)$, $Y_{D}(M)$, and $Y_{R}(M)$ be the number of links, delay, and data rate of session $S$ respectively. The number of links used in the mapped Multilayer Hyper-Graph for session $S$ is the summation of all binary numbers in $M$. Hence, $Y_{L}(M)$ is given by

$$
Y_{L}(M)=\sum_{\forall p_{t} \in S_{t}, \forall q_{r} \in S_{r} \backslash p_{r}} \sum_{\forall(i, j) \in V} M_{i j}^{p_{t} q_{r}}
$$

The delay of the Many-to-Many session is the longest delay among all paths in the Many-to-Many session, which is given by

$$
\begin{aligned}
& Y_{D}(M)=\operatorname{Max}\left(\sum_{\forall(i, j) \in V} M_{i j}^{p_{t} q_{r}} d_{i j}\right), \\
& \forall p_{t} \in S_{t}, \forall q_{r} \in S_{r} \backslash p_{r}
\end{aligned}
$$

where $d_{i j}$ is the delay between node $i$ and node $j . d_{i j}$ includes transmission and switching delay between $i$ and $j$.
The rate of the transmission between node $i$ and node $j$, $r_{i j}$, is the rate of the node with the minimum rate. Hence, $r_{i j}=\operatorname{Min}\left(r_{i}, r_{j}\right)$, where $r_{i}$ and $r_{j}$ are the rates of nodes $i$ and $j$ respectively. To find the rate of the Many-to-Many session $S$, we need to find the rate of the link with the minimum rate, which is given by

$$
\begin{aligned}
& Y_{R}(M)=\operatorname{Min}\left(M_{i j}^{p_{t} q_{r}} r_{i j}+\left(1-M_{i j}^{p_{t} q_{r}}\right) K\right), \\
& \forall p_{t} \in S_{t}, \forall q_{r} \in S_{r} \backslash p_{r}, \forall(i, j) \in V .
\end{aligned}
$$

where $K$ is a very large number.

Let $\vec{Y}(M)$ be the objective function vector, which is given by

$$
\vec{Y}(M)=\left(Y_{L}(M), Y_{D}(M), Y_{R}(M)\right) .
$$

Hence, the multi-objective optimization problem can be formulated as follows:

$$
\underset{M}{\operatorname{Minimize}}: \vec{Y}_{m m}(M)=\left(Y_{L}(M), Y_{D}(M),-Y_{R}(M)\right)
$$

\section{Subject to:}

$$
\begin{aligned}
& \sum_{\forall(i, x) \in V} M_{i x}^{p_{t} q_{r}}-\sum_{\forall(x, j) \in V} M_{x j}^{p_{t} q_{r}}=\left\{\begin{array}{l}
1, \quad \text { if } x=q_{r} \\
-1, \quad \text { if } x=p_{t} \\
0, \quad \text { otherwise }
\end{array}\right. \\
& \forall p_{t} \in S_{t}, \forall q_{r} \in S_{r} \backslash p_{r}
\end{aligned}
$$

$$
\begin{gathered}
\sum_{\forall p_{t} \in S_{t}, \forall q_{r} \in S_{r} \backslash p_{r}} M_{i j}^{p_{t} q_{r}} \leq 1 \quad \forall i, j \in V \\
M_{i j}^{p_{t} q_{r}}+M_{j i}^{p_{t} q_{r}} \leq 1 \quad \forall p_{t} \in S_{t}, \forall q_{r} \in S_{r} \backslash p_{r}, \forall i, j \in V \\
Y_{D}(M) \leq d_{\max } \\
r_{\min } \leq Y_{R}(M) \quad \forall p_{t} \in S_{t}, \forall q_{r} \in S_{r} \backslash p_{r} \\
Y_{L}(M), Y_{D}(M)>0 \quad \forall p_{t} \in S_{t}, \forall q_{r} \in S_{r} \backslash p_{r} \\
M_{i j}^{p_{t} q_{r}} \in\{0,1\} \quad \forall p_{t} \in S_{t}, \forall q_{r} \in S_{r} \backslash p_{r}, \forall i, j \in V
\end{gathered}
$$

In this optimization problem, (9) is the routing constraint for the flow in each link in the Many-to-Many session, (10) ensures that each link between any couple of nodes is used for routing a flow destined for only one path from a source to a destination and (11) ensures that the transmission between any couple of nodes is in one direction. The constraint in (12) is an upper bound on $Y_{D}(M)$, where $d_{\max }$ is the maximum allowable delay. The constraints in (13-14) are lower bounds on $Y_{L}(M), Y_{D}(M)$ and $Y_{R}(M)$, where $r_{\min }$ is the required bit rate for the path between node $p_{t}$ and node $q_{r}$, and (15) indicates that $M_{i j}^{p_{t} q_{r}}$ is a binary number.

The optimization problem in (8-15) is an Integer Liner Program (ILP) problem. The decision variables of this problem are $M_{i j}^{p_{t} q_{r}}$, where the parameters are $K, d_{i j}, d_{\text {max }}, r_{\text {min }}$, and $r_{i}, \forall p_{t} \in S_{t}, \forall q_{r} \in S_{r} \backslash p_{r}, \forall i, j \in V$. 
TABLE I: Notations

\begin{tabular}{cl}
\hline \hline Notation & Definition \\
\hline & \\
$S N_{i}$ & Super Node i. \\
$s\left(S N_{i}\right)$ & Source SU of $S N_{i}$. \\
$d\left(S N_{i}\right)$ & A set of destination SUs of $S N_{i}$. \\
$T_{i}$ & Many-to-Many subgraph initiated by $S U i$. \\
\hline
\end{tabular}

\section{SChEdULing OF SNs' Transmission}

In wireless networks, simultaneous transmissions may cause interference as a result of sharing the wireless medium. Hence, random access MAC protocols or transmission scheduling need to be utilized in order to avoid transmission collision. In this paper, we schedule the transmissions of the SNs over a cycle $\mathrm{T}$, where $\mathrm{T}$ is slotted into several synchronized slots. An SN's transmission may conflict with another SN's transmission due to reasons other than interference. For example, two overlapping SNs may conflict with each other due to the assumption that each $\mathrm{SU}$ has a single radio and can not transmit and receive at the same time.

In this section, the possible conflicts between SNs (or Hyperedges) is studied. In each SN, one SU is responsible for transmission to all other SUs belonging to that SN. This transmission must be scheduled in such a way that no conflict happens between any two or more transmitting SUs. A conflict between any two SNs means that they cannot be scheduled for transmission in the same time slot.

The reasons for conflict between SNs can be summarized as follows:

1) Let $S U B$ transmit to $S U D$ over channel h. According to the protocol interference model in [6], $S U D$ can successfully receive transmissions from $S U B$ if

$$
\left|L_{S U_{z}}-L_{S U D}\right| \geq(1+\delta)\left|L_{S U B}-L_{S U D}\right|
$$

where $S U_{z}$ is any simultaneously transmitting SU over the same channel other than $S U B$, and $\delta$ is a positive parameter. Let $S N_{i}$ and $S N_{j}$ belong to one layer and $s\left(S N_{k}\right) \in d\left(S N_{j}\right)$. If the source of $S N_{k}, s\left(S N_{k}\right)$, is located within the interference range of $S N_{i}$ 's source, $s\left(S N_{i}\right)$, then there is a conflict between $S N_{i}$ and $S N_{j}$. In Figure 4 (a), $S U D$ is located within the interference range of $S U A$, and $S U D \in d\left(S N_{2}\right)$. Therefore, there is a conflict between $S N_{1}$ (HE 1) and $S N_{2}$ (HE 2).

2) If $s\left(S N_{j}\right) \in d\left(S N_{i}\right)$, and both $S N_{i}$ and $S N_{j}$ are destined for routing the traffic initiated by one Manyto-Many session's source, then $S N_{i}$ conflicts with $S N_{j}$. If $s\left(S N_{i}\right)=s\left(S N_{j}\right)$, then there is no conflict between $S N_{j}$ and $S N_{i}$ when they are destined for routing the traffic initiated by one SU. Figure 4 (b) shows that the source of $S N_{2}, S U B$, is also a destination for SU A in $S N_{1}$. Hence, $S N_{1}$ and $S N_{2}$ cannot be scheduled for transmission at the same time slot. If $S N_{2}$ operates on different channel as in Figure 4 (c), the conflict between $S N_{1}$ and $S N_{2}$ is still valid since the above condition is met, and each node uses a single transceiver.

3) If $s\left(S N_{i}\right)=s\left(S N_{j}\right)$, and $S N_{i}$ and $S N_{j}$ are destined for routing the traffic initiated by different Many-to-Many session's sources, then $S N_{i}$ conflicts with $S N_{j}$. Figure 4 (d) shows that there is a conflict between $S N_{1}$ and $S N_{3}$ since $s\left(S N_{1}\right)=s\left(S N_{3}\right)$ and both $S N_{1}$ and $S N_{3}$ are destined for different routing trees.

4) If $S N_{i}$ and $S N_{j}$ are on different layers, and $s\left(S N_{i}\right)=$ $s\left(S N_{j}\right)$, then there is a conflict in transmission schedule between $S N_{i}$ and $S N_{j}$. For example, $S U A$ is the source for $S N_{1}$ and $S N_{3}$ as shown in Figure 4 (e). $S U A$ cannot transmit on different channels at the same time while having only a single radio. Hence, $S N_{1}$ and $S N_{3}$ must be scheduled for transmission in different time slots.

5) Let $S N_{i}$ and $S N_{k}$ belong to one layer, $S N_{j}$ and $S N_{m}$ belong to another layer, $s\left(S N_{k}\right) \in d\left(S N_{i}\right)$ and $s\left(S N_{m}\right) \in d\left(S N_{j}\right)$. if $s\left(S N_{k}\right)=s\left(S N_{m}\right)$, then there is a conflict between $s\left(S N_{i}\right)$ and $s\left(S N_{j}\right)$. In Figure 4 (f), $S N_{1}$ conflicts with $S N_{3}$ because the condition above is satisfied.
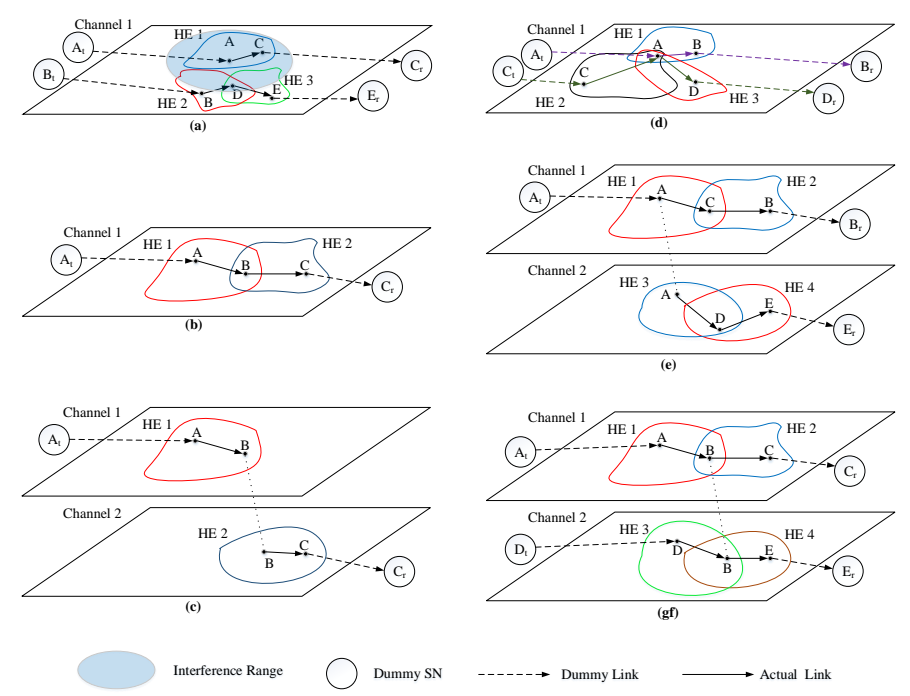

Fig. 4. Conflicts Between SNs.

After finding all pairs of conflicting SNs, we construct the conflict graph $G_{C}$ of the original graph $G(V, E)$. By using the Weighted coloring Algorithm in [7] while considering the weighted SNs instead of weighted links, we can find the schedule of the transmission for each SN. Therefore, all nonconflicting SNs can transmit in one time slot. The SN with a lower data rate requires more time slots than the $\mathrm{SN}$ with a higher data rate. To calculate the weight of each SN, the SN with the highest rate is assigned weight 1 and all other SNs are weighted by $\left\lceil\frac{\text { Max Rate }}{\mathrm{SN} \text { 's Rate }}\right\rceil[8]$.

\section{Solving the Multi-OBJective Optimization fOR MANY-TO-MANY COMMUNICATION USING ANT COLONY OPTIMIZATION}

In Section III, we modeled the optimization problem as an ILP since the problem is NP-Hard [12]. To obtain a solution of the problem in polynomial time, we use the Ant colony optimization (ACO) [9] meta-heuristic approach to solve our problem. ACO was inspired by the behavior of ants when 
they search for a food source. The ants deposit pheromone along their path from their nest to the target source. Since the pheromone evaporates over time, the shortest path to the source will have the strongest pheromone concentration. Therefore, future ants tend to select shorter paths.

In this paper, we use ACO to solve the multiobjective optimization problem for Many-to-Many communication in CRNs. Given a set $S$, which represents a many-to-many session, each $S U_{l} \in S$ establishes a subtree $T_{l}$ that reaches all other $S U_{m} \in S \backslash S U_{l}$. The union of all subtrees forms a manyto-many communication subgraph $T_{m 2 m}$, and the optimization is performed over this union. Each $S U_{l} \in S$ is associated with a pheromone matrix $\tau^{l}$ such that each matrix is updated independently. $\tau^{l}=\cup \tau_{i j}^{l}, \forall(i, j) \in E$, where $\tau_{i j}^{l}$ is the pheromone intensity on the link between $S N_{i}$ and $S N j$ observed by the ants during the establishment of subtree $T_{l}$. For each subtree $T_{l} \in T_{m 2 m}$, the probability that ant $k$ in $S N_{i}$ chooses to move to $S N_{j}$ is given by [9]

$$
P_{i j}^{k}(l)= \begin{cases}\frac{\left[\tau_{i j}^{l}\right]^{\alpha} \eta_{i j}^{\beta}}{\sum_{\forall q \in Z_{k}}\left[\tau_{i q}^{l}\right]^{\alpha} \eta_{i q}^{\beta}} & \text { if } j \in Z_{k}, \\ 0 & \text { Otherwise. }\end{cases}
$$

where $Z_{k}$ is a set of all neighboring nodes unvisited by ant $k$, and $\alpha$ and $\beta$ are parameters used to weight $\tau_{i j}^{l}$ and $\eta_{i j}$ respectively. $\eta_{i j}$ is the visibility to $S N_{j}$ when the ant is on $S N_{i}$, which is given by

$$
\eta_{i j}=\frac{1}{T_{x}^{j}+T_{s w}^{i j}}
$$

where $T_{x}^{j}$ is the transmission time inside supernode $j$, and $T_{s w}^{i j}$ is the switching time between the channels corresponding to supernode $i$ and supernode $j$. For an ant in supernode $i$, a higher visibility to supernode $j$ means lower delay for the ant to transit to supernode $j$.

We use a modified version of the Multi-objective Ant Colony Optimization Algorithms (MOACS) in [10] and [8] to find the Pareto optimal set as described in Algorithm 1. A solution is called Pareto optimal if it is not dominated by any other solutions found by MOACS. The input of the algorithm is a mapped Hyper-Graph and a Many-to-Many session request $S$. The session request $S$ is a set of SUs denoted by $\left\{X_{1}, X_{2}, \ldots, X_{|S|}\right\}$, where each SU sends its messages to all other SUs in $S$. The output of Algorithm 1 are many-to-many subgraph, where each many-to-many subgraph $T_{m 2 m}$ provides a Pareto optimal solution in terms of delay, rate and number of links.

Algorithm 1 starts by initializing $T_{m 2 m}, Y_{s o l}$ and $\tau_{m n}^{l}$, where $Y_{s o l}$ is a set of all $T_{m 2 m}$ subgraphs and $\tau_{0}$ is the initial pheromone value. In lines 3-7, $|S|$ sub-trees are established from each source to all other sources. In lines 8-9, the transmissions of all $S N s$ in $T_{m 2 m}$ are scheduled, and the number of links, delay and rate are calculated. The Algorithm in lines 10-11 dismisses the solution if it is not feasible. In lines $12-13, Y_{\text {sol }}$ is updated to remove any solution dominated by $T_{m 2 m}$. If the recently generated $T_{m 2 m}$ is not dominated by any other Many-to-Many tree, the pheromone matrices are reinitialized as shown in lines 14-15. This reinitialization helps in finding another solution in the following iterations,

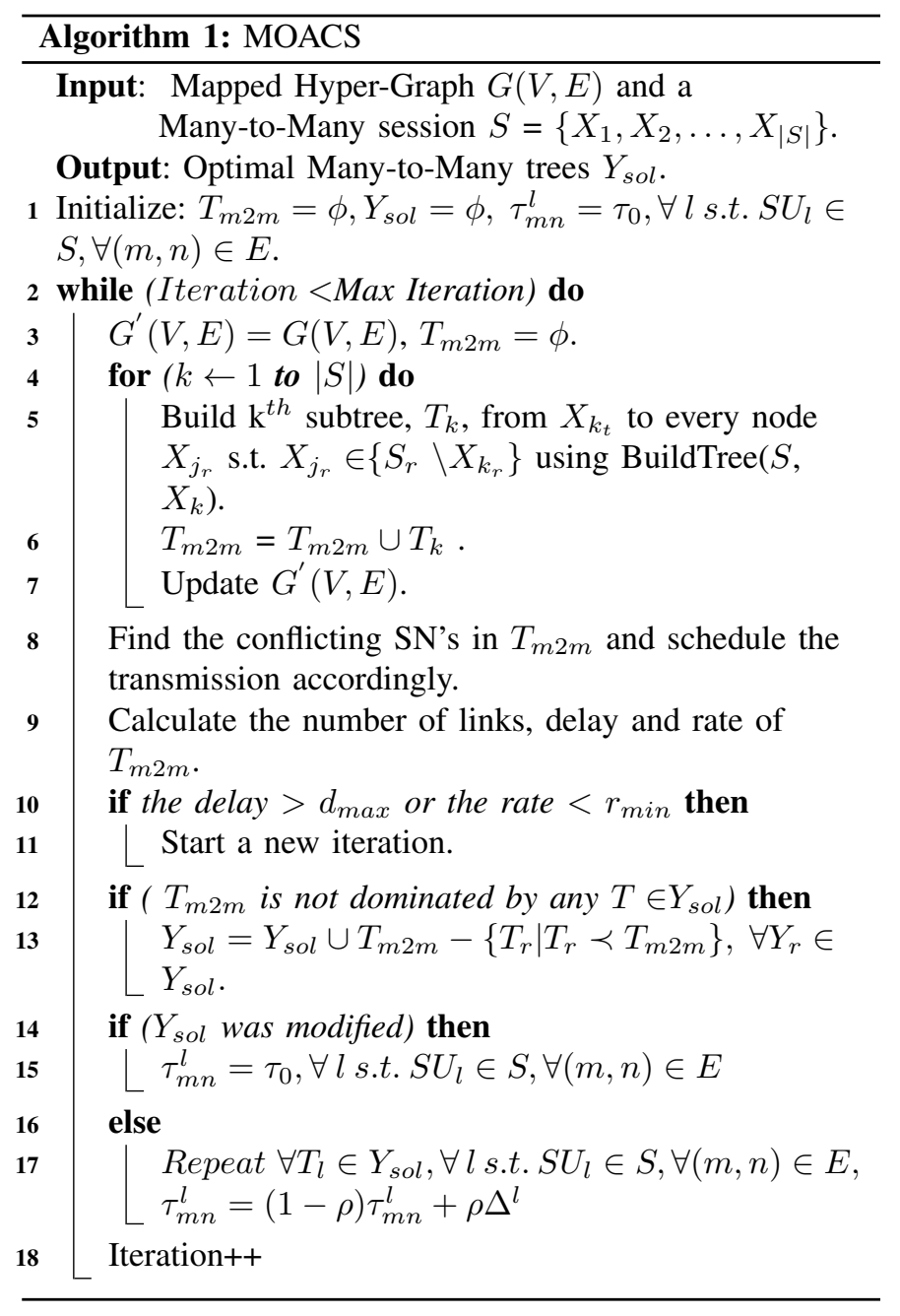

which may not be dominated by any tree in $Y_{s o l}$. If $T_{m 2 m}$ is dominated, then $\tau_{m n}^{l}$ is updated as shown in lines 16-17 to exploit all already found solutions to find a better solution in the next iterations, where $\rho$ is the pheromone evaporation rate, and $\Delta^{l}$ is given by

$$
\Delta^{l}=\frac{1}{w_{1} \text { Delay }_{l}+w_{2} \text { NumOfLinks }_{l}}+w_{3} \text { Rate }_{l} .
$$

where $w_{1}, w_{2}$ and $w_{3}$ are weighting factors, and Delayl, NumOfLinks $s_{l}$, and Rate R $_{l}$ are the delay the number of links and the rate of $T_{l}$ respectively. The while loop continues until the maximum number of iterations is reached.

\section{Vi. Simulation Results}

In this section, we present simulation results for multiobjective optimization for many-to-many communication in a CRN. We consider a CRN with 6 channels and $50 \mathrm{SUs}$ distributed over $300 \mathrm{~m}$ by $300 \mathrm{~m}$ area. Transmission range of each $S U$ can be $120,130,140,150,160$ or $170 \mathrm{~m}$. The $S U s$ located within the transmission range of each other form an $S N$, where the rate of the $S N$ is calculated using equation 1 . Table II shows the parameters used in the simulation.

Using Algorithm 1, we find the optimal Pareto set. Then, we compare the performance of the multi-ojective optimization algorithm given by Algorithm 1 with a single-objective 

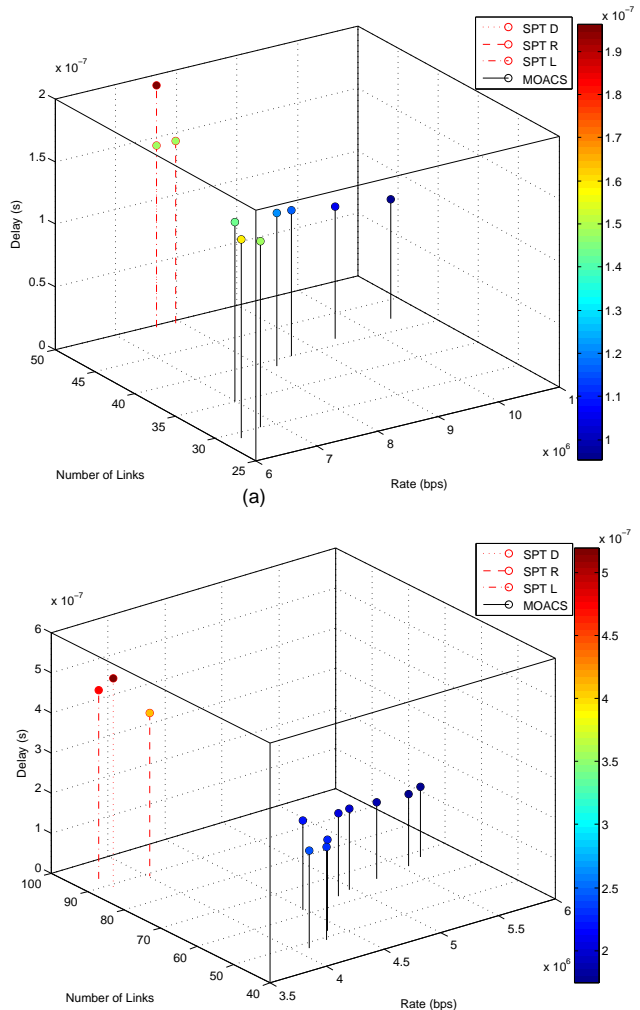

(b)

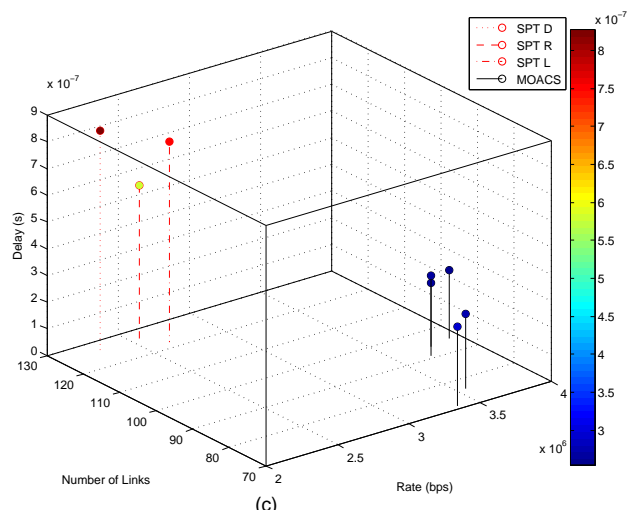

Fig. 5. The performance of the Algorithm 1 and SPT when: (a): group size is 4 , (b): group size is 5 , (c) group size is 6 .

TABLE II: Simulation Parameters

\begin{tabular}{|c|c|c|c|}
\hline Parameter & Value \\
\hline \hline$W$ & $6 \mathrm{MHz}$ \\
\hline$P$ & $0.1 \mathrm{~W}$ \\
\hline$g$ & 1 \\
\hline$\epsilon$ & 2 \\
\hline$N_{0}$ & $W / \mathrm{Hz}$ \\
\hline$\delta$ & 0.1 \\
\hline
\end{tabular} \begin{tabular}{|cc} 
Parameter & Value \\
\hline$d_{\max }$ & $1 \mu \mathrm{s}$ \\
\hline$r_{\min }$ & $1 \mathrm{Mbps}$ \\
\hline$P_{P U_{i}}$ & $0.1, \forall i \in\{1, \ldots, 6\}$ \\
\hline Packet Size & 1500 Bytes \\
\hline Group Size & $4-6$ \\
\hline
\end{tabular}

algorithm that finds the shortest-path tree (SPT). In the singleobjective algorithm, a shortest path tree is generated from each source $S N$ to all destination $S N^{\prime} s$ using Dijkstra's algorithm. The shortest-path tree is an approximation to the minimum Steiner tree. We consider each of the delay, rate and number of links as single objectives for finding the shortest path tree in terms of delay, rate and number of links, respectively.

In the simulation, we generate a random CRN according to the parameters mentioned earlier. Then, we generate 5 random sessions for a specific group size. Fig.5 shows comparisons between the performance of Algorithm 1 (MOACS) with the single-objective algorithms (SPT D, SPT R and SPT L) when the group size is 4,5 and 6 , respectively. SPT D, SPT R and SPT L represent SPT when the single objective is the delay, rate and number of links, respectively.

As the group size increases, the delay and the number of links increases and the date rate decreases. It is shown in Fig.5 that the solutions of MOACS are not dominated by either SPT D, SPT R or SPT L. In other words, the solutions generated by MOACS are better at least in one objective. Most of MOACS solutions outperform the solutions obtained by SPT D, SPT $\mathrm{R}$ and SPT L in all objectives. For example, four solutions found by MOACS dominate the solutions of SPT D, SPT R and SPT L algorithm when the group size is 4 . Moreover, all MOACSC solutions dominate the solutions of SPT D, SPT R and SPT L when the group size is 5 or 6 .

\section{CONCLUSION}

We studied the routing and scheduling problem for manyto-many communication in CRNs while optimizing the QoS of the SUs. We modeled the optimization problem as an ILP, and solved it using an Ant Colony meta-heuristic. The Pareto front found by MOACS was found to outperforms single-objective SPT algorithm in delay, rate and the number of transmission links.

\section{REFERENCES}

[1] S. L. Hakimi, Steiners problem in graphs and its implications, Networks, vol. 1, pp. 113133, 1971.

[2] M. R. Garey, R. L. Graham, and D. S. Johnson, The complexity of computing steiner minimal trees, SIAM J. Appl. Math., vol. 32, no. 4, pp. 835859, June 1977.

[3] Alnabelsi, S. and A. E. Kamal, " Resilient Multicast Routing in CRNs Using a Multilayer Hyper-graph Approach", in the proceedings of the IEEE International Conference on Communications (ICC) conference, 2013.

[4] G. Cheng, W. Liu, Y. Li, and W. Cheng, Spectrum aware on-demand routing in cognitive radio networks, in Proc. 2nd IEEE Int. Symp. New Frontiers in Dynamic Spectrum Access Netw. (DySpan), 2007, pp. 571574.

[5] S. Shin, K. Lee, and S. M. Kang, 4.2mW CMOS frequency synthesizer for $2.4 \mathrm{GHz}$ ZigBee application with fast settling time performance, IEEE Int. Microwave Theory and Tech. Symp., pp. 411-414, 2006.

[6] P. Gupta and P. R. Kumar, The capacity of wireless networks, IEEE Trans. Inform. Theory, vol. 46, no. 2, pp. 388404, 2000.

[7] W. Wang, Y. Wang, X. Y. Li, W. Z. Song, and O. Frieder, Efficient interference-aware TDMA link scheduling for static wireless networks, in Proc. of ACM MobiCom, 2006.

[8] Jie, Y. and A. E. Kamal, "Multi-Objective Multicast Routing Optimization in Cognitive Radio Networks", in the proceedings of the IEEE Wireless Communications and Networking Conferences (WCNC) 2014.

[9] Dorigo M, "Optimization, learning and natural algorithms". $\mathrm{PhD}$ thesis, Dipartimento di Elettronica, Politecnico di Milano, Italy, 1992 [in Italian].

[10] D. Pinto and B. Baran, "Solving Multiobjective Multicast Routing Problem with a New Ant Colony Optimization Approach", in 2nd IFIP/ACM Latin American Networking Conference 2005 - LANC'05, Cali, Colombia, pp. 11-19, October 2005.

[11] M. Dorigo, M. Birattari, and T. Stutzle, Ant Colony Optimization Artificial Ants as a Computational Intelligence Technique, IEEE Comput. Intell. Mag., vol. 1, no. 4, pp. 2839, 2006.

[12] M. R. Garey and D. S. Johnson, Computers and Intractability: A Guide to the Theory of NP-Completeness, W. H. Freeman, 1979. 